\title{
Acute Mitral Regurgitation Following Myocardial Infarction
}

\author{
Drew Johnson, MD and Colin Craft, MD
}

\section{CASE REPORT}

A 66-year-old Caucasian female with no past medical history presented with four hours of dyspnea. The patient stated that two days prior to presentation she developed severe, crushing substernal chest pain that awoke her from sleep. The pain was non-radiating, lasted 30 minutes, and was associated with diaphoresis, shortness of breath, nausea, and lightheadedness. Her medical history was unremarkable except for a 50-pack-year smoking history.

On presentation, the patient was tachycardic to 110 beats per minute, and tachypnic at 22 breaths per minute. Cardiac auscultation revealed a 4/6 holosystolic murmur radiating to the axilla that was audible throughout the precordium. Pulmonary exam revealed rales at the bases bilaterally. JVP was elevated to the angle of the mandible. Though she was initially able to breathe comfortably and speak in full sentences, over the next hour she developed increased work of breathing and respiratory distress

The patient's admission labs were notable for an elevated troponin $T$ of $1.54 \mathrm{ng} / \mathrm{mL}$. Electrocardiogram (EKG) revealed sinus tachycardia, with ST elevations in leads II, III, and aVf. Chest X-ray showed mild pulmonary edema. Bedside echocardiography revealed segmental wall motion abnormalities with basal to mid inferior akinesis. There was a ruptured posteromedial papillary muscle and a flail mitral valve leaflet with severe mitral regurgitation. Flow reversal was seen in the pulmonary arteries. Ejection fraction was 70\% +/- 5 .

Soon after presentation, the patient became hypotensive and hypoxic, and required intubation. She had developed cardiogenic shock due to an inferior ST segment elevation myocardial infarction (STEMI) complicated by a ruptured papillary muscle, likely due to delayed presentation to the hospital. She underwent emergent cardiac catheterization, which revealed $100 \%$ occlusion of the mid-right coronary artery. Intra-aortic balloon pump (IABP) was placed due to cardiogenic shock. The patient then underwent an emergent mitral valve replacement. The procedure was complicated by pericardial hematoma, and wound infection. Unfortunately, the patient developed cardiorespiratory failure, requiring venoarterial extracorporeal membrane oxygenation. The patient passed away after a prolonged hospitalization in the intensive care unit.

\section{INTRODUCTION}

In the United States, myocardial infarction (MI) is a major cause of mortality. While the vast majority of patients suffering an acute MI present early in the disease course, those who present later are at greater risk for mechanical complications. Risks associated with MI are not only immediate risk of myocardial muscle damage due to the impaired coronary blood flow, but also mechanical complications due to weakening of the structural integrity of the heart, such as rupture of the ventricular free wall, rupture of the interventricular septum, and acute mitral regurgitation from a ruptured chordae or papillary muscle. While these mechanical complications are rare, they can have devastating effects and often present as life-threatening emergencies. This report reviews the current literature regarding acute mitral regurgitation and subsequent papillary muscle rupture following a STEMI.

\section{BACKGROUND}

Mechanical complications of MI have become much less common with the development and increased availability of percutaneous coronary intervention (PCl) and fibrinolytics. This is because more rapid reperfusion of coronary blood flow limits the myocardial damage. There are three main mechanical complications that occur following myocardial infarction:

1. acute mitral regurgitation from a ruptured chordae or papillary muscle,

2. rupture of the interventricular septum, and

3. rupture of the free ventricular wall. The incidence is less than $1 \%$ for each of these mechanical complications.

Given the emergent nature of these complications, immediate diagnosis and treatment are needed to reduce morbidity and mortality of these conditions. Prior to the fibrinolytic era, mechanical complications occurred later in the disease course. Rupture of the interventricular septum is currently observed in the first 24 hours post MI, whereas before fibrinolytics, it was seen up to a week after MI. Rupture of the papillary muscle is now most commonly diagnosed within 13 hours as shown in the SHOCK trial registry, while previously, it was observed two to seven days post MI. 
Both pre- and post- fibrinolytic era, rupture of the ventricular free wall is most commonly observed within five to 14 days. ${ }^{3}$

There are multiple pathophysiologic causes of acute mitral regurgitation including myocardial wall aneurysm, left ventricular dilation, chordae rupture, and rupture of a papillary muscle. ${ }^{4}$ Mitral regurgitation can be seen in $13-45 \%$ of patients after an acute MI. While this is usually transient, if the cause is a ruptured papillary muscle, it can persist. Of patients presenting with cardiogenic shock, about $7 \%$ have a papillary muscle rupture as the cause. This contributes $5 \%$ of the mortality after acute MI, and is associated with poor clinical outcome.4-7 Acute ischemic mitral regurgitation presents most commonly in patients with inferior wall MI, with the posteromedial papillary muscle being six to twelve times more commonly involved than the anterolateral wall. This is because the posterior descending artery provides the solitary arterial supply to the posteromedial papillary muscle. Alternatively, the anterolateral papillary muscle is supplied by the left anterior descending and the circumflex arteries. ${ }^{3}$

\section{DIAGNOSIS}

Diagnosis is based largely on a high clinical suspicion, history, and physical examination, and is often aided by the use of echocardiography and ventriculography. History typically includes severe chest pain, however there can be atypical presentations. Atypical presentations occur more commonly in elderly individuals, women, and diabetics where dyspnea may be the chief complaint. The clinical signs are commonly shortness of breath, tachypnea, chest pain, rales on pulmonary auscultation, and a loud murmur consistent with mitral regurgitation, often with radiation to the axilla. The murmur of mitral regurgitation can also be silent due to the decreased ventricular function decreasing the regurgitant flow velocity, thereby limiting the atrioventricular pressure gradient and causing a reduced murmur. ${ }^{8}$ The intensity of the murmur does not correlate with the amount of regurgitation. As time passes, patients may develop respiratory distress, hypotension, and hypoxia. EKG is likely to show inferior STEMI, and chest X-ray may show bilateral pulmonary edema or focal right upper lobe pulmonary edema due to the mitral regurgitation jet directed toward the right pulmonary arteries. Factors that increase the risk of a mechanical complication include post infarct angina, delayed hospitalization greater than 24 hours, and undue in-hospital physical activity. ${ }^{9}$

\section{MANAGEMENT}

Once diagnosed, the treatment of choice for ischemic papillary muscle rupture is surgical valve replacement or repair due to the risk for rapid decompensation. Dobutamine, an inotrope that increases cardiac contractility and heart rate by acting on beta1, beta2 and alpha1 adrenergic receptors, may be used to increase cardiac output. An IABP can also be placed in order to stabilize the patient until surgical intervention. ${ }^{10}$ IABPs deflate during systole to help increase cardiac output, and inflate during diastole to increase coronary blood flow and diastolic pressure, resulting in an increase in cardiac output by $20 \%$. Both interventions aim to increase cardiac output until a more permanent intervention can be performed. Despite the multitude of medical interventions available, surgical correction remains the cornerstone of ischemic papillary muscle rupture treatment. Currently, complete valve replacement is preferred over valve repair, but research suggests that valve repair may result in improved outcomes. ${ }^{11}$ Additional research is needed to determine the long-term outcomes and durability of mitral valve repair in the treatment of papillary muscle rupture. While the mortality rates have been estimated at $27 \%$ perioperatively, surgery remains the best option for patients. ${ }^{1,12}$ The mortality rate for patients who do not undergo operative intervention is around $50 \%$ in the first 24 hours, and increases with time. This reinforces the importance of prompt revascularization for patients with acute myocardial infarctions in order to prevent mechanical complications. Unfortunately, even if the complications are realized early, they still carry a high mortality rate as seen in the case described.

\section{KEY POINTS}

Mechanical complications of myocardial infarctions are uncommon today due to increased availability of coronary reperfusion. Major mechanical complications include:

1. rupture of the free ventricular wall,

2. rupture of the interventricular septum, and

3. acute mitral regurgitation from a ruptured chordae or papillary muscle.

Diagnosis hinges on effective history and physical examination, and interpretation of EKG, chest radiography, and echocardiography. Although it carries high mortality rate, timely diagnosis is essential in order to initiate potentially life-saving therapies early, in order to improve outcomes. Treatment of acute mitral regurgitation secondary to papillary muscle rupture includes coronary reperfusion, temporizing measures such as IABP, and afterload reduction until definitive surgical management with valve replacement or repair. 


\section{REFERENCES}

1. Kutty, Ramesh S., Nicola Jones, and Narain Moorjani. "Mechanical Complications of Acute Myocardial Infarction." Cardiology Clinics 31.4 (2013): 519-31. Web.

2. Antman, Elliott M. "ST-Elevation Myocardial Infarction." The AHA Guidelines and Scientific Statements Handbook (n.d.): 46-90.

3. Grasso, Adam, and Sorin Brener. "Complications Of Acute Myocardial Infarction." Complications of Acute Myocardial Infarction. Cleveland Clinic, July 2014. Web. 05 Mar. 2017.

4. Reeder, Guy S. "Identification and Treatment of Complications of Myocardial Infarction." Mayo Clinic Proceedings 70.9 (1995): 880-84.

5. Hillis GS, Møller JE, Pellikka PA, Bell MR, Casaclang-Verzosa GC, Oh JK. Prognostic significance of echocardiographically defined mitral regurgitation early after acute myocardial infarction. Am Heart J 2005; 150:1268-1275.

6. Davis N, Sistino JJ. Review of ventricular rupture: key concepts and diagnostic tools for success. Perfusion 2002; 17:63-67.

7. Hochman, Judith S., Christopher E. Buller, Lynn A. Sleeper, Jean Boland, Vladimir Dzavik, Timothy A. Sanborn, Emilie Godfrey, Harvey D. White, John Lim, and Thierry Lejemtel. "Cardiogenic Shock Complicating Acute Myocardial Infarction etiologies, Management and Outcome: A Report from the SHOCK Trial Registry." Journal of the American College of Cardiology 36.3 (2000): 1063-070. Web.
8. Bursi, F. "Heart Failure and Death After Myocardial Infarction in the Community: The Emerging Role of Mitral Regurgitation." Circulation 111.3 (2005): 295-301. Web

9. Figueras, Jaume, Josefa Cortadellas, Francisco Calvo, and Jordi Soler-Soler. "Relevance of Delayed Hospital Admission on Development of Cardiac Rupture during Acute Myocardial Infarction: Study in 225 Patients with Free Wall, Septal or Papillary Muscle Rupture." Journal of the American College of Cardiology 32.1 (1998): 135-39.

10. Kishon, Yehezkiel, Jae K. Oh, Hartzell V. Schaff, Charles J. Mullany, A. Jamil Tajik, and Bernard J. Gersh. "Mitral Valve Operation in Postinfarction Rupture of a Papillary Muscle: Immediate Results and Long-Term Follow-Up of 22 Patients." Mayo Clinic Proceedings 67.11 (1992): 1023-030.

11. Lee, Sung Kwang, Woon Heo, Ho-Ki Min, Do Kyun Kang, Hee Jae Jun, and Youn-Ho Hwang. "A New Surgical Repair Technique for Ischemic Total Papillary Muscle Rupture." The Annals of Thoracic Surgery 100.5 (2015): 1891-893. Web.

12. Thompson CR, Buller CE, Sleeper LA, et al; for the SHOCK Investigators. Cardiogenic shock due to acute severe mitral regurgitation complicating acute myocardial infarction: a report from the SHOCK Trial Registry. J Am Coll Cardiol 2000; 36(3 suppl A):1104-1109.

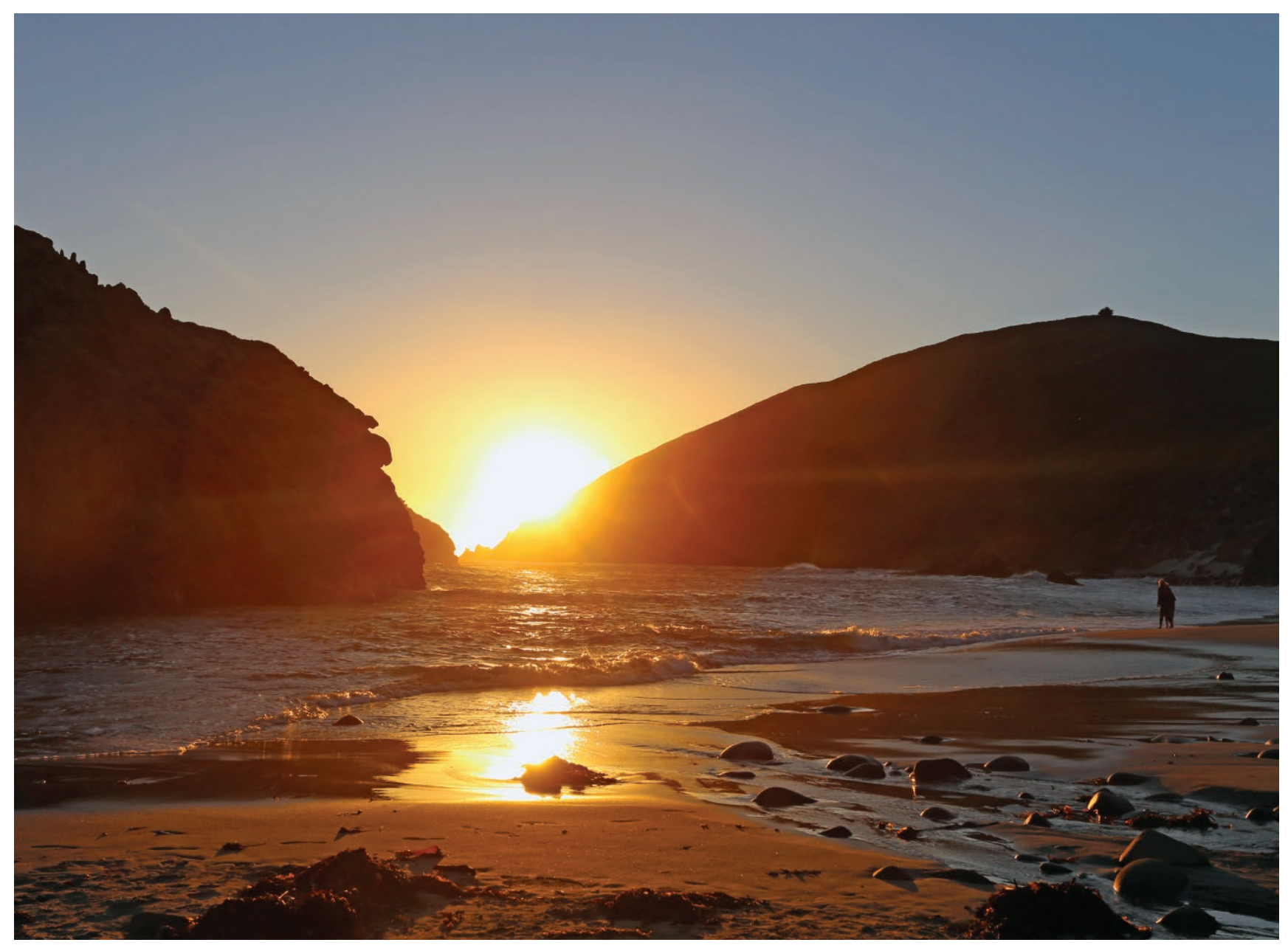

Sharon Li, MD 